\title{
New-Onset Atrial Fibrillation in Patients With Coronavirus Disease 2019 (COVID-19) and Cardiovascular Disease - Insights From the CLAVIS-COVID Registry -
}

\author{
Takahide Sano, MD; Shingo Matsumoto, MD, PhD; Takanori Ikeda, MD, PhD; \\ Shunsuke Kuroda, MD; Takeshi Kitai, MD, PhD; Taishi Yonetsu, MD, PhD; \\ Shun Kohsaka, MD, PhD; Sho Torii, MD, PhD; \\ Koichi Node, MD, PhD; Yuya Matsue, MD, PhD
}

\begin{abstract}
Background: Both pre-existing atrial fibrillation (AF) and new-onset AF (NOAF) are observed in patients with coronavirus disease 2019 (COVID-19); however, the effect of AF on clinical outcomes is unclear. This study aimed to investigate the effect of AF, especially NOAF, on the outcomes of hospitalized patients with COVID-19.
\end{abstract}

\begin{abstract}
Methods and Results: This study analyzed 673 COVID-19 patients with cardiovascular diseases and risk factors (CVDRF). Patients were divided into 3 groups; pre-existing AF $(n=55)$, NOAF $(n=28)$, and sinus rhythm (SR) $(n=590)$. The baseline characteristics and in-hospital outcomes were evaluated. The mean age of the patients was 68 years, $65.4 \%$ were male, and the in-hospital mortality rate was $15.6 \%$. The NOAF group demonstrated a higher in-hospital mortality rate $(42.9 \%)$ than the pre-existing AF (30.9\%) and SR $(11.2 \%)$ groups $(\mathrm{P}<0.001)$. Patients with NOAF had a higher incidence of acute respiratory syndrome, multiple organ disease, hemorrhage, and stroke than those with pre-existing AF and NOAF. NOAF was independently associated with in-hospital mortality after adjusting for pre-existing AF and 4C mortality score (odds ratio [95\% confidence interval]: 4.71 [1.63-13.6], $\mathrm{P}<0.001$ ).
\end{abstract}

Conclusions: Patients with NOAF had significantly worse outcomes as compared to patients with pre-existing AF and SR. The incidence of NOAF would be a useful predictor of clinical outcomes during hospitalization.

Key Words: Atrial fibrillation; Cardiovascular diseases; COVID-19; Japan; Mortality

$\mathbf{P}$ re-existing cardiovascular diseases and risk factors (CVDRF), including atrial fibrillation (AF), are commonly observed and associated with higher mortality rates after infection with severe acute respiratory syndrome coronavirus 2 (SARS-CoV-2), the causative virus of coronavirus disease 2019 (COVID-19). ${ }^{\mathbf{1} 2}$ SARS$\mathrm{CoV}-2$ specifically impairs the cardiovascular system and causes myocardial injury, which is related to worse clinical outcomes during hospitalization, particularly in those with underlying cardiovascular disease (CVD) ${ }^{3-5}$ These facts highlight the need to focus on underlying and new-onset CVDs during the clinical course of SARS-CoV-2 infection as a remarkable and independent predictor of outcomes, which can add clinical value to existing prediction models of COVID-19. Given that the prediction of the clinical course in patients with COVID-19 is difficult due to their heterogeneity, the $4 \mathrm{C}$ mortality score, ${ }^{6,7}$ which is another useful predictor independent from the pre-existing prediction model developed and validated specifically in patients with COVID-19, is warranted.

$\mathrm{AF}$ is a common arrhythmia that increases the risk of morbidity and mortality in patients with CVD. ${ }^{8,9}$ The incidence of new-onset atrial fibrillation (NOAF) in patients with COVID-19 is common, and is related to worse out-

Received November 2, 2021; revised manuscript received November 19, 2021; accepted December 13, 2021; J-STAGE Advance Publication released online January 26, 2022 Time for primary review: 16 days

Department of Cardiovascular Medicine, Toho University Graduate School of Medicine, Tokyo (T.S., T.I.); Division of Cardiovascular Medicine, Department of Internal Medicine, Toho University Faculty of Medicine, Tokyo (S.M.), Japan; Department of Cardiovascular Medicine, Cleveland Clinic, OH (S. Kuroda), USA; Department of Cardiovascular Medicine, National Cerebral and Cardiovascular Center, Suita (T.K.); Department of Cardiovascular Medicine, Tokyo Medical and Dental University, Tokyo (T.Y.); Department of Cardiology, Keio University School of Medicine, Tokyo (S. Kohsaka); Department of Cardiology, Tokai University School of Medicine, Isehara (S.T.); Department of Cardiovascular Medicine, Saga University, Saga (K.N.); and Department of Cardiovascular Biology and Medicine (Y.M.), Cardiovascular Respiratory Sleep Medicine (Y.M.), Juntendo University Graduate School of Medicine, Tokyo, Japan

The first two authors contributed equally to this work (T.S., S.M.).

Mailing address: Shingo Matsumoto, MD, PhD, Division of Cardiovascular Medicine, Department of Internal Medicine, Toho University Faculty of Medicine, 6-11-1 Omorinishi, Ota-ku, Tokyo 143-8541, Japan. E-mail: shingomatsumoto0606@gmail.com

All rights are reserved to the Japanese Circulation Society. For permissions, please e-mail: cj@j-circ.or.jp

ISSN-1346-9843 


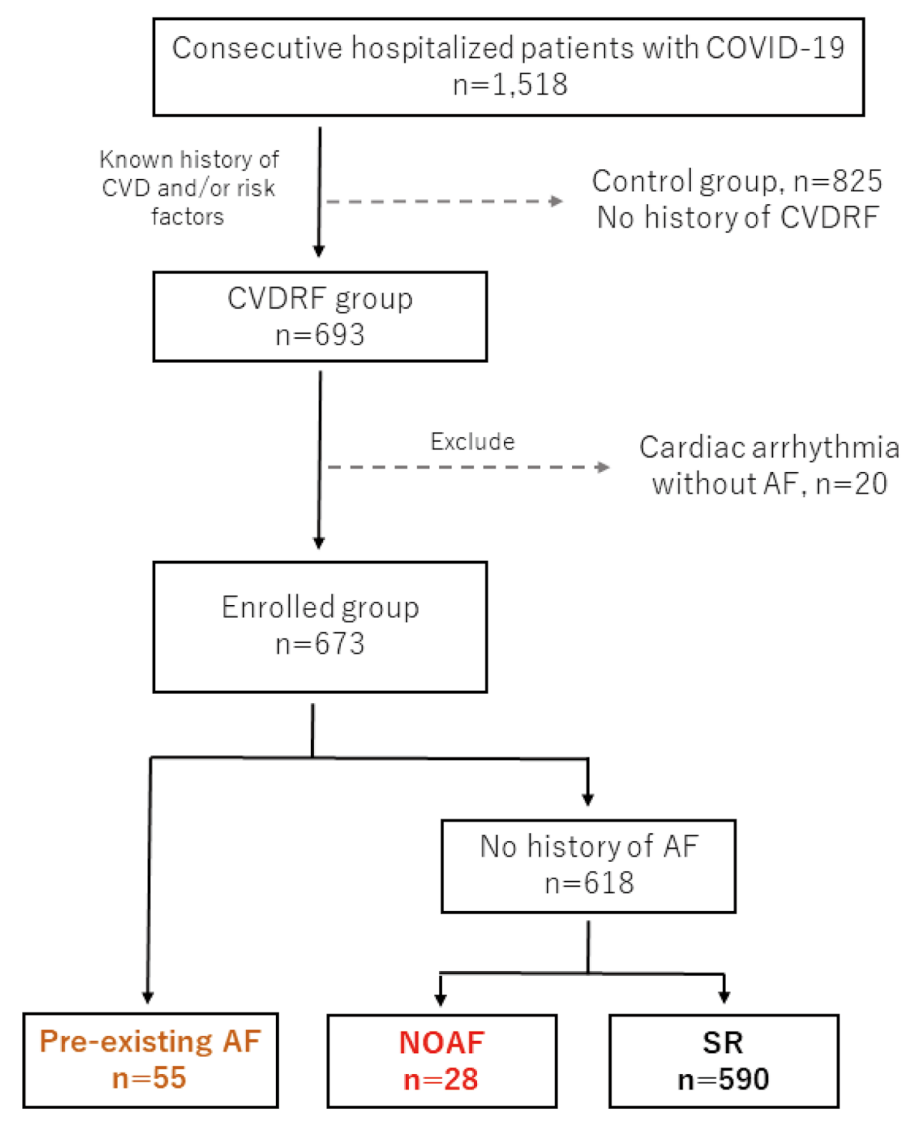

Figure 1. Study flow chart. AF, atrial fibrillation; COVID-19, coronavirus disease 2019; CVD, cardiovascular diseases; CVDRF, cardiovascular diseases and risk factors; NOAF, new-onset atrial fibrillation; $\mathrm{SR}$, sinus rhythm.

comes after SARS-CoV-2 infection. ${ }^{10,11}$ Previous reports have suggested that mechanisms of SARS-CoV-2 infection are related to the occurrence of myocardial injury, 12,13 acute myocardial infarction, ${ }^{12}$ heart failure, 12,14,15 arrhythmia, ${ }^{10,12,16}$ and thrombotic events, ${ }^{12,17,18}$ hence, the incidence of AF after SARS-CoV-2 infection is assumed to be observed in the general population. Indeed, recent studies have suggested that patients with COVID-19 have a considerable risk of NOAF during the clinical course. ${ }^{19,20}$ Considering severe inflammation status is closely associated with the incidence of $\mathrm{AF}$, the relationship between COVID-19 infection and NOAF may result from the severity of systemic inflammation subsequent to the infection. Thus, we hypothesized that NOAF is a promising independent predictor of clinical outcomes in patients with COVID-19, which adds value to existing prediction models. However, most studies evaluating the effect of NOAF on clinical outcomes of COVID-19 have had small sample sizes and, therefore, could not analyze the independency toward other established prediction models; thus, the generalizability of these results remains controversial.

The clinical outcomes of SARS-CoV-2 infection in hospitalized patients with cardiovascular diseases and/or risk factors (CLAVIS-COVID) registry, a Japanese nationwide study sponsored by the Japanese Circulation Society, was designed to evaluate outcomes in patients with COVID-19 and CVDRF as a specific subtype of COVID-19 associated with poor outcomes after infection. ${ }^{21-23}$ In this post-hoc analysis of CLAVIS-COVID registry, we aimed to investigate the effect of AF, especially NOAF, on the clinical outcomes of hospitalized patients with COVID-19.

\section{Study Design and Population}

The CLAVIS-COVID registry was designed to evaluate the clinical features and outcomes of patients with pre-existing CVDRF and COVID-19 hospitalized between 1 January and 31 May 2020 in 49 acute care hospitals, and was sponsored by the Japanese Circulation Society. ${ }^{24}$ SARSCoV-2 infection was diagnosed on the basis of a positive polymerase chain reaction (PCR) test of a nasal or pharyngeal swab specimen. Patients aged $<20$ years at the time of admission were excluded from this study. CVDRF involves CVDs and cardiovascular risk factors, such as hypertension, diabetes mellitus, and dyslipidemia. Cardiovascular diseases were defined as heart failure, coronary artery disease, myocardial infarction, peripheral artery disease, valvular heart disease, cardiac arrhythmia, pericarditis, myocarditis, congenital heart disease, pulmonary hypertension, deep vein thrombosis, pulmonary embolism, aortic dissection, aortic aneurysm, cerebral infarction/transient ischemic attack, use of cardiac devices (e.g., a pacemaker, implantable cardioverter defibrillation, cardiac resynchronization therapy, left ventricular assist device), heart transplantation, and cardiac arrest.

In the present study, 693 patients had COVID-19 and an underlying CVDRF. To compare patients with AF and sinus rhythm (SR), those with a history of cardiac arrythmia without AF, such as sick sinus syndrome, atrial-ventricular 


\begin{tabular}{|c|c|c|c|c|c|}
\hline Variables & $\begin{array}{c}\text { Number of } \\
\text { data }\end{array}$ & $\begin{array}{c}\text { Pre-existing AF } \\
\qquad(\mathrm{n}=55)\end{array}$ & $\begin{array}{l}\text { NOAF } \\
(n=28)\end{array}$ & $\begin{array}{c}S R \\
(n=590)\end{array}$ & $P$ value \\
\hline Age (years) $)^{\star, \star *}$ & 673 & $77.3 \pm 10.1$ & $75.8 \pm 12.8$ & $66.9 \pm 14.8$ & $<0.001$ \\
\hline Male & 673 & $36(65.5)$ & $17(60.1)$ & $387(65.6)$ & 0.89 \\
\hline BMI $\left(\mathrm{kg} / \mathrm{m}^{2}\right)$ & 560 & $23.5 \pm 5.6$ & $23.5 \pm 4.6$ & $24.4 \pm 5.0$ & 0.33 \\
\hline Smoker/ex-smoker & 637 & $19(36.5)$ & $15(55.6)$ & $231(41.4)$ & 0.27 \\
\hline Hypertension ${ }^{\star *}$ & 673 & $31(56.4)$ & $22(84.6)$ & $450(76.3)$ & 0.007 \\
\hline Diabetes mellites & 673 & $17(30.9)$ & $8(28.6)$ & $235(39.8)$ & 0.25 \\
\hline Dyslipidemia & 673 & $18(32.7)$ & $9(32.1)$ & $236(40)$ & 0.46 \\
\hline Heart failure ${ }^{\star *, \star * \star}$ & 673 & $20(36.4)$ & $1(3.6)$ & $32(5.4)$ & $<0.001$ \\
\hline Ischemic heart disease ${ }^{* *}$ & 673 & $13(23.6)$ & $6(21.4)$ & $61(10.3)$ & 0.0047 \\
\hline $\mathrm{Cl} / \mathrm{TI}^{\star \star *, \star \star *}$ & 673 & $13(23.6)$ & $1(3.6)$ & $37(6.3)$ & $<0.001$ \\
\hline Chronic lung disease & 673 & $6(10.9)$ & $3(10.7)$ & $55(9.3)$ & 0.78 \\
\hline$C K D^{\star \star, \star \star \star}$ & 673 & $10(18.1)$ & $1(3.6)$ & $34(5.8)$ & 0.005 \\
\hline Cancer & 673 & $7(12.7)$ & $5(17.9)$ & $53(9.0)$ & 0.17 \\
\hline $\mathrm{CHA}_{2} \mathrm{DS}_{2}-\mathrm{VASC}^{\star \star, \star \star *}$ & 673 & $3.82(3-5)$ & $3.11(2-4)$ & $2.66(2-4)$ & $<0.001$ \\
\hline \multicolumn{6}{|l|}{ Baseline medication } \\
\hline ACE-I/ARB*** & 673 & $14(25.5)$ & $15(53.6)$ & $227(38.5)$ & 0.036 \\
\hline$\beta$-blocker ${ }^{*, \star *}$ & 673 & $20(36.4)$ & $8(28.6)$ & $74(12.5)$ & $<0.001$ \\
\hline CCB & 673 & $14(25.5)$ & $10(35.7)$ & $233(39.5)$ & 0.12 \\
\hline Diuretics $^{* *}$ & 673 & $15(27.3)$ & $3(10.7)$ & $39(6.6)$ & $<0.001$ \\
\hline Statin & 673 & $14(25.5)$ & $10(35.7)$ & $183(31.0)$ & 0.62 \\
\hline 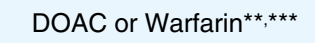 & 673 & $33(60)$ & $1(3.6)$ & $12(2.0)$ & $<0.001$ \\
\hline \multicolumn{6}{|l|}{ Vital signs } \\
\hline Glasgow coma scale & 606 & $15(15-15)$ & $15(15-15)$ & $15(15-15)$ & 0.294 \\
\hline Heart rate (beats/min) & 667 & $87.2 \pm 20.7$ & $84.1 \pm 19.7$ & $86.7 \pm 17.4$ & 0.74 \\
\hline Systolic BP $(\mathrm{mmHg})^{\star \star}$ & 669 & $126.6 \pm 21$ & $127 \pm 20.5$ & $134.1 \pm 21.7$ & 0.015 \\
\hline Diastolic BP $(\mathrm{mmHg})^{\star *}$ & 668 & $74.9 \pm 14.9$ & $73.1 \pm 15.2$ & $79.3 \pm 15.4$ & 0.02 \\
\hline Respiratory rate & 523 & $20.2 \pm 5.4$ & $19.2 \pm 5.2$ & $21.1 \pm 6.2$ & 0.27 \\
\hline $\mathrm{SpO}_{2}(\%)$ & 669 & $95.6 \pm 3.2$ & $93.2 \pm 7.9$ & $94.4 \pm 4.7$ & 0.09 \\
\hline \multicolumn{6}{|l|}{ Symptoms } \\
\hline Cough & 673 & $20(36.4)$ & $15(53.6)$ & $289(49.0)$ & 0.17 \\
\hline Sore throat & 673 & $2(3.6)$ & $1(3.6)$ & $69(11.7)$ & 0.10 \\
\hline Fatigue & 673 & $19(36.5)$ & $10(35.8)$ & $196(33.2)$ & 0.93 \\
\hline Panting/Dyspnea & 673 & $19(36.5)$ & $12(42.9)$ & $188(31.9)$ & 0.42 \\
\hline Dysgeusia & 673 & $3(5.5)$ & $0(0)$ & $42(7.1)$ & 0.46 \\
\hline Asymptomatic & 673 & $4(7.3)$ & $3(10.7)$ & $37(6.3)$ & 0.52 \\
\hline HF edema* & 673 & $7(12.7)$ & $2(7.1)$ & $8(1.4)$ & $<0.001$ \\
\hline HF congestion** & 673 & $10(18.2)$ & $3(10.7)$ & $23(3.9)$ & $<0.001$ \\
\hline
\end{tabular}

Values are expressed as $\mathrm{n}(\%)$ or mean \pm standard deviation, median (interquartile range). ${ }^{*}$ Significant difference in the comparison of the 2 groups, NOAF and SR. ${ }^{*}$ Significant difference in the comparison of the 2 groups, pre-existing AF and SR. ${ }^{* * *}$ Significant difference in the comparison of the 2 groups, pre-existing AF and NOAF. ACE-I, angiotensin-converting enzyme inhibitor; AF, atrial fibrillation; ARB, angiotensin 2 receptor blocker; $\mathrm{BMI}$, body mass index; $\mathrm{BP}$, blood pressure; $\mathrm{CCB}$, calcium channel blocker; $\mathrm{Cl}$, cerebral infarction; $\mathrm{CKD}$, chronic kidney disease; COVID-19, coronavirus disease 2019; DOAC, direct oral anticoagulants; HF, heart failure; NOAF, new-onset atrial fibrillation; SpO2, peripheral oxygen saturation; SR, sinus rhythm; TIA, transient ischemic attack.

block, ventricular tachycardia, and other arrythmias, and those with a history of pacemaker implantation were excluded. Finally, 673 patients were enrolled and divided into 3 groups: pre-existing AF, NOAF, and SR (Figure 1). NOAF was defined as new-onset AF after admission, which was sustained for $>24 \mathrm{~h}$ in patients without pre-existing AF. Pre-existing AF involved a history of AF, including paroxysmal, persistent, and permanent AF, and manifestations of AF on admission. The differences in characteristics and in-hospital outcomes among the 3 groups were analyzed. The primary endpoint was in-hospital mortality.

The investigation conformed to the principles outlined in the Declaration of Helsinki. The study protocol, including the use of an opt-out consent method, was approved by the ethics committee of Toho University Omori Medical Center (No. M20253, 20089, 20040, 20017) and the local ethics committees of all participating institutions. This clinical study was registered with the University Hospital Medical Information Network Clinical Trial Registry (UMIN-ID: UMIN000040598) before the first patient was enrolled, in accordance with the International Committee of Medical Journal Editors.

\section{Data Collection}

Our dataset referred to the case report form proposed by the International Severe Acute Respiratory and Emerging 
Table 2. Examination Findings at the Time of Admission and Treatment During Hospital Course $(n=673)$

\begin{tabular}{|c|c|c|c|c|c|}
\hline & $\begin{array}{l}\text { Number of } \\
\text { data }\end{array}$ & $\begin{array}{l}\text { Pre-existing AF } \\
\qquad(n=55)\end{array}$ & $\begin{array}{l}\text { NOAF } \\
(n=28)\end{array}$ & $\begin{array}{c}\text { SR } \\
(n=590)\end{array}$ & $P$ value \\
\hline \multicolumn{6}{|l|}{ Laboratory data } \\
\hline CRP (mg/L) $)^{\star}$ & 647 & $5.8(3.8-11.4)$ & $9.2(5.8-15.1)$ & $5.8(1.8-11.5)$ & 0.017 \\
\hline WBC count & 658 & $5,790(1,700-7,450)$ & $6,050(4,505-7,932)$ & $5,700(4,400-7,560)$ & 0.81 \\
\hline Neutrophil count $(\%)^{*}$ & 583 & $75.8(66.5-83.7)$ & 79.8 (73.6-83.8) & 74.6 (64.4-82.3) & 0.08 \\
\hline Hemoglobin level $(\mathrm{g} / \mathrm{dL})^{\star *}$ & 659 & $12.2(10.9-13.9)$ & $13(11.8-13.9)$ & $13.6(11.9-14.8)$ & 0.001 \\
\hline Hematocrit $(\%)^{*}$ & 647 & $37.1(33.3-41.5)$ & $37.8(35.7-41.3)$ & $40(35.5-43.4)$ & 0.008 \\
\hline $\operatorname{PLT}\left(10^{4} / \mu \mathrm{L}\right)^{*}$ & 656 & $16.8(12.4-25.1)$ & $16.1(11.6-22.4)$ & $19.1(15.1-24.8)$ & 0.03 \\
\hline Albumin $(\mathrm{g} / \mathrm{dL})^{\star *}$ & 620 & $2.9(2.5-3.5)$ & $3.1(2.9-3.4)$ & $3.3(2.9-3.8)$ & $<0.001$ \\
\hline $\mathrm{Na}(\mathrm{mEq} / \mathrm{L})$ & 659 & $137(135-139)$ & $137(135-140)$ & $138(135-141)$ & 0.77 \\
\hline $\mathrm{K}(\mathrm{mEq} / \mathrm{L})$ & 659 & $4.1(3.7-4.4)$ & $3.8(3.6-4.2)$ & $4(3.7-4.3)$ & 0.25 \\
\hline eGFR $\left(\mathrm{mL} / \mathrm{min} / 1.73 \mathrm{~m}^{2}\right)^{\star *}$ & 660 & $73.7(55.8-94.7)$ & $73.6(45.9-103.3)$ & $88.5(67.0-106.7)$ & 0.002 \\
\hline Blood urea nitrogen (mmol/L) & 660 & $6.2(5.4-9.7)$ & $5.7(5.0-8.9)$ & $5.9(4.3-8.6)$ & 0.90 \\
\hline $\mathrm{LDH}(\mathrm{IU} / \mathrm{L})$ & 598 & $290(211-409)$ & $364(261-433)$ & $291(227-413)$ & 0.20 \\
\hline D-dimer (ng/mL) & 448 & $1.53(0.78-2.62)$ & $1.5(1.10-1.97)$ & $1.4(0.71-3.02)$ & 0.98 \\
\hline Troponin positive & 138 & $10(55.6)$ & $6(75)$ & $54(48.2)$ & 0.34 \\
\hline BNP $(p g / m L)^{\star \star \star \star *}$ & 211 & $375.2(164.0-749.5)$ & $94.6(43.5-216.5)$ & $26.3(10.1-83.9)$ & $<0.001$ \\
\hline \multicolumn{6}{|l|}{ Chest X-ray } \\
\hline $\begin{array}{l}\text { GGO/consolidation/alveolar } \\
\text { opacity, }, \star \star \star \star\end{array}$ & 611 & $41(50.9)$ & $27(96.4)$ & $386(65.4)$ & 0.008 \\
\hline Congestion ${ }^{\star, \star *}$ & 611 & $15(23.3)$ & $6(21.4)$ & $29(5.5)$ & $<0.001$ \\
\hline Pleural effusion ${ }^{\star, \star *}$ & 611 & $10(18.9)$ & $5(17.9)$ & $28(4.7)$ & $<0.001$ \\
\hline \multicolumn{6}{|l|}{ Chest CT } \\
\hline GGO/consolidation & 526 & $39(84.8)$ & $21(87.5)$ & $347(76.1)$ & 0.24 \\
\hline Pleural effusion ${ }^{\star, \star *}$ & 526 & $12(26.1)$ & $10(41.7)$ & $50(11.0)$ & $<0.001$ \\
\hline \multicolumn{6}{|l|}{ Treatment } \\
\hline Heparin ${ }^{\star, \star \star *}$ & 673 & $14(25.5)$ & $15(53.6)$ & $111(18.8)$ & $<0.001$ \\
\hline Catecholamine & 673 & $10(18.2)$ & $8(28.6)$ & $80(13.6)$ & 0.071 \\
\hline Inotropic drugs & 673 & $3(5.5)$ & $2(7.1)$ & $24(4.1)$ & 0.42 \\
\hline Vasopressor* & 673 & $7(12.7)$ & $8(28.6)$ & $64(10.8)$ & 0.03 \\
\hline $\mathrm{O}_{2}$ demand $^{\star, \star \star}$ & 670 & $41(74.5)$ & $27(96.4)$ & $365(62.2)$ & $<0.001$ \\
\hline ICU entry ${ }^{\star, \star \star}$ & 673 & $18(32.7)$ & $17(60.7)$ & $162(27.5)$ & 0.001 \\
\hline Intubation/respiration ${ }^{\star, \star \star}$ & 673 & $13(23.6)$ & $14(50)$ & $124(21)$ & 0.004 \\
\hline Renal replacement therapy & 673 & $2(3.6)$ & $3(10.7)$ & $39(6.6)$ & 0.49 \\
\hline $\begin{array}{l}\text { New introduction of renal } \\
\text { replacement therapy }\end{array}$ & 673 & $1(1.8)$ & $2(7.1)$ & $22(3.7)$ & 0.41 \\
\hline V-A ECMO & 673 & $0(0)$ & $1(3.6)$ & $2(0.34)$ & 0.14 \\
\hline V-V ECMO & 673 & $0(0)$ & $2(7.1)$ & $21(3.6)$ & 0.14 \\
\hline
\end{tabular}

Values are expressed as $\mathrm{n}(\%)$ or mean \pm standard deviation, median (interquartile range). ${ }^{*}$ Significant difference in the comparison of the 2 groups, NOAF and SR. ${ }^{*}$ Significant difference in the comparison of the 2 groups, pre-existing AF and SR. ${ }^{* *}$ Significant difference in the comparison of the 2 groups, pre-existing AF and NOAF. BNP, B-type natriuretic peptide; CRP, C-reactive protein; CT, computed tomography; eGFR, estimated glomerular filtration rate; GGO, ground-grass opacity; ICU, intensive care unit; LDH, lactate dehydrogenase; PLT, platelet; V-A ECMO, vena-arterial extracorporeal membrane oxygenation; V-V ECMO, veno-venous extracorporeal membrane oxygenation; WBC, white blood cell. Other abbreviations as in Table 1.

Infection Consortium. ${ }^{25}$

Clinical characteristics, including symptoms, demographics, medical history, home medications, baseline comorbidities, physical findings, laboratory test results, radiography and chest computed tomography (CT) findings, electrocardiography and cardiac echocardiography results, treatment information, and outcomes, were obtained from the patients' electronic medical records using data collection forms. All baseline laboratory and imaging data were obtained at the time of admission. In the analysis of cardiac troponin (cTn), a positive result was defined as an elevation above the $99^{\text {th }}$ percentile of the upper reference limit value in each assay. The date of COVID-19 onset was defined as the first day of symptom onset; otherwise, if the patients had no symptoms on admission, the day of the first positive PCR test for SARS-CoV-2 was considered.

During the period of patient enrollment, the Japanese government mandated the hospitalization of all patients diagnosed with COVID-19 based on the result of the PCR test, regardless of severity. The participating hospitals fundamentally followed this recommendation. The discharge threshold of all patients with COVID-19 was also determined by the Japanese government guidelines for COVID-19 management. ${ }^{26}$ The guidelines recommend that patients remain in hospital until: (1) their systemic conditions and respiratory symptoms are improved and stable; 


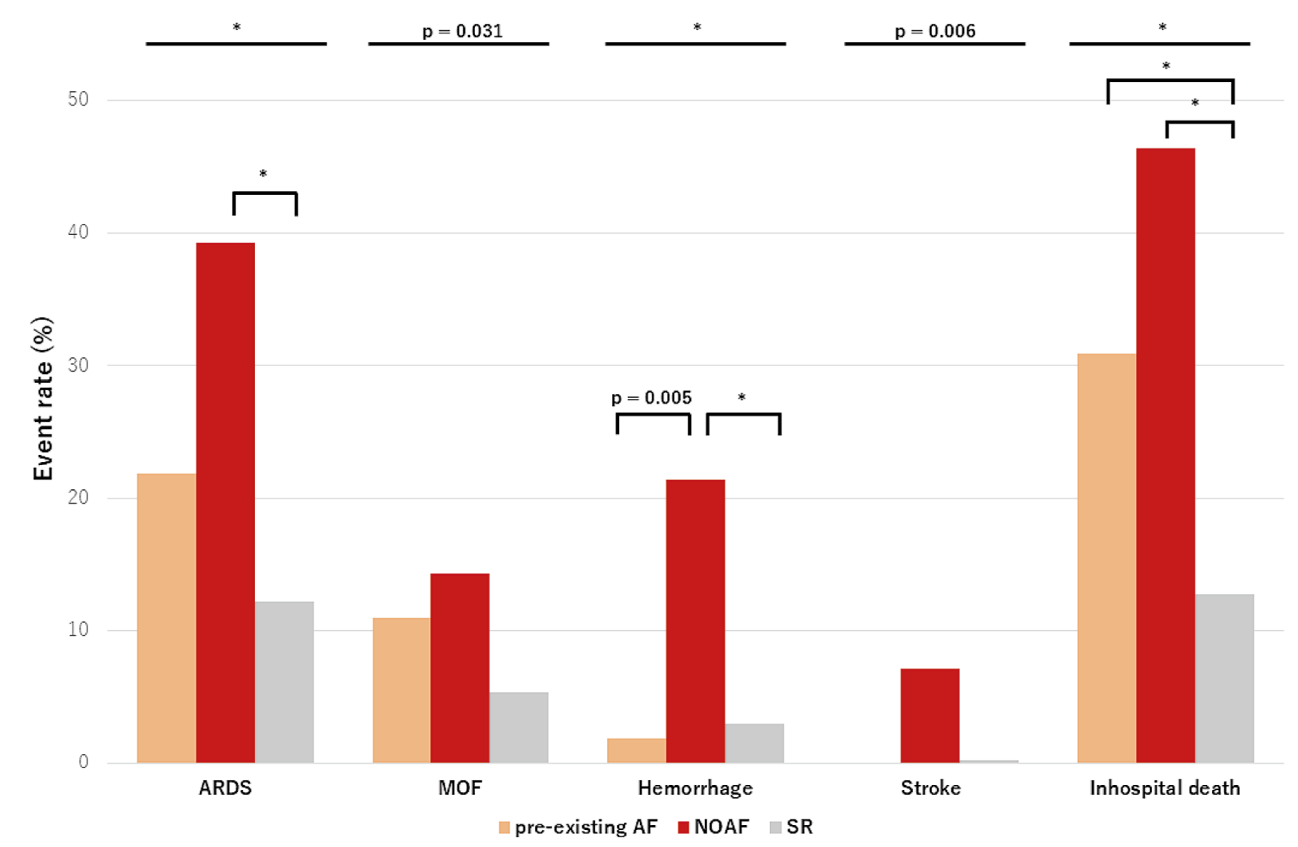

Figure 2. In-hospital outcomes in patients with coronavirus disease 2019 (COVID-19; pre-existing AF, NOAF, and SR groups). ${ }^{*} \mathrm{P}<0.001$. AF, atrial fibrillation; ARDS, acute respiratory syndrome; MOF, multiple organ failure; NOAF, new-onset atrial fibrillation; SR, sinus rhythm.

(2) their body temperature is consistently $<37.5^{\circ} \mathrm{C}$, for at least $24 \mathrm{~h}$; and (3) negative results are confirmed twice, at least $12 \mathrm{~h}$ apart.

\section{Statistical Analysis}

All data are described as presenting frequency, percentages $(\%)$ for categorical variables, and the mean value ( \pm standard deviation) or median value with interquartile range (quartiles 1-3) for continuous variables. Fisher's exact test was used to evaluate categorical variables, and the Kruskal-Wallis test was used to assess continuous variables. Univariate and multivariate logistic regression analysis models were used to estimate the effect of pre-existing AF and NOAF on in-hospital outcomes. We used the 4C mortality score, developed and validated in patients with COVID-19, for the adjustment in the multivariable logistic model.6,7 The model validation in our cohort has already been described and performed elsewhere. ${ }^{27}$ All analyses in the present study were performed using Stata software version 15 (StataCorp) and R (version 4.0.3).

\section{Results}

\section{Patient Characteristics}

Among the 673 patients, the mean age was 68 years, and $65.4 \%$ were men. They were categorized into pre-existing $\mathrm{AF}$ (55 patients), NOAF (28 patients), and SR (590 patients) groups (Figure 1).

Table 1 presents the baseline characteristics of patients stratified by AF status. Patients with pre-existing AF and NOAF were significantly older than those with SR. History of heart failure, cerebral vascular diseases, and chronic renal disease were more common in patients with pre-existing AF than in the other groups. In addition, the pre-existing
AF group was associated with a higher $\mathrm{CHA}_{2} \mathrm{DS}_{2}-\mathrm{VASc}$ score.

The use of $\beta$-blockers and diuretics as well as history of heart failure were significantly more common in the preexisting AF group than in the NOAF and SR groups. In contrast, treatment with an angiotensin-converting enzyme inhibitor or angiotensin 2 receptor blocker was significantly more frequent in patients with NOAF compared with that for other groups. New oral anticoagulants or warfarin are frequently used in patients with preexisting AF.

\section{Symptoms and Vital Signs}

Overall, the most common symptoms at the time of admission were cough $(48.1 \%)$, fatigue $(33.4 \%)$, and dyspnea $(32.5 \%)$. There was no significant difference in the symptoms according to AF status. The pre-existing AF group had a higher incidence of heart failure symptoms, such as edema and congestion, than the NOAF and SR groups. The SR group had significantly higher systolic and diastolic blood pressure at the time of admission than the other groups. However, the heart rate, respiratory rate, and peripheral oxygen saturation on admission were not significantly different between the 3 groups.

\section{Laboratory and Imaging Findings}

The baseline laboratory and imaging findings are shown in Table 2. Although there were no significant differences in the number of white blood cells and neutrophils among the 3 groups, the levels of C-reactive protein (CRP) and lactate dehydrogenase $(\mathrm{LDH})$ were significantly higher in patients with NOAF than in those of the other groups. Moreover, baseline hemoglobin, hematocrit, platelet, and albumin levels were significantly higher in patients with SR than in those with pre-existing AF and NOAF. The estimated 


\begin{tabular}{|cccccc|}
\hline \multicolumn{2}{|c|}{ Table 3. 4C Mortality Scores at the Time of Admission $(\mathbf{n}=\mathbf{4 6 1 )}$} & & & \\
Variable & $\begin{array}{c}\text { Overall } \\
(\mathbf{n}=\mathbf{4 6 1 )}\end{array}$ & $\begin{array}{c}\text { Pre-existing AF } \\
(\mathbf{n}=\mathbf{3 8})\end{array}$ & $\begin{array}{c}\text { NOAF } \\
(\mathbf{n}=\mathbf{2 1})\end{array}$ & $\begin{array}{c}\text { SR } \\
(\mathbf{n}=\mathbf{4 0 2})\end{array}$ & P value \\
4C mortality score & $8.4 \pm 3.8$ & $10.1 \pm 2.9$ & $10.0 \pm 3.7$ & $8.2 \pm 3.8$ & 0.001 \\
\hline
\end{tabular}

Values are expressed as mean \pm standard deviation. Abbreviations as in Table 1.

\begin{tabular}{|c|c|c|c|}
\hline Variable & OR & $95 \% \mathrm{Cl}$ & $P$ value \\
\hline NOAF & 4.71 & $1.63-13.6$ & 0.004 \\
\hline Pre-existing AF & 2.09 & $0.90-4.82$ & 0.09 \\
\hline 4C mortality score & 1.44 & $1.31-1.59$ & $<0.001$ \\
\hline
\end{tabular}

OR, odds ratio; $\mathrm{Cl}$, confidence interval. Other abbreviations as in Table 1.

glomerular filtration rate was also significantly higher in patients with SR. Moreover, the B-type natriuretic peptide level in the NOAF group was significantly higher than that in the SR group, but less than that in the pre-existing AF group. The D-dimer level and proportion of positive cTn (i.e., $>99$ percentile upper reference limit) were not statistically different among the 3 groups.

Regarding chest X-ray on admission, findings of ground grass opacity/consolidation/alveolar opacity and congestion were more frequently observed in the NOAF group than in the other groups. Patients with NOAF and preexisting AF had a higher incidence of congestion on chest radiography and pleural effusion on chest radiography and $\mathrm{CT}$, with a significant difference compared to those with SR.

\section{Treatment During Hospital Course}

The use of antibiotics, heparin, and vasopressors was significantly more frequent in patients with NOAF than in the other groups (Table 2). Furthermore, the rates of oxygenation and respiratory ventilator use were significantly higher in patients with NOAF.

\section{In-Hospital Outcomes}

In-hospital adverse events, including the incidence of acute respiratory distress syndrome (ARDS), sepsis, septic shock, stroke, acute kidney injury, liver disorder, multiple organ failure, and hemorrhage, were more common in patients with NOAF than in those with pre-existing AF and SR. The number of patients treated in the intensive care unit after admission was also significantly higher in the NOAF group than in the other groups (Figure 2).

The overall in-hospital mortality was $15.6 \%$, and the in-hospital all-cause mortality was significantly different according to the AF status. Patients with NOAF had significantly higher mortality than those with pre-existing $\mathrm{AF}$ or SR (pre-existing AF vs. NOAF vs. SR groups: $30.9 \%$ vs. $46.4 \%$ vs. $12.7 \%, \mathrm{P}<0.001$ ) (Figure 2). Furthermore, NOAF was independently associated with in-hospital mortality even after adjustment for pre-existing $\mathrm{AF}$ and the $4 \mathrm{C}$ mortality score (odds ratio [95\% confidence interval]: 4.71 [1.63-13.6], $\mathrm{P}=0.004)$ (Tables 3,4).

\section{Discussion}

In the current study, the presence of both NOAF and preexisting AF led to higher in-hospital mortality than SR after SARS-CoV-2 infection. In addition, we found that patients with NOAF had worse in-hospital adverse events, such as multiple organ disease, hemorrhage, stroke, and in-hospital death; this was even in comparison to those with pre-existing $\mathrm{AF}$, despite a higher rate of underlying heart failure and chronic kidney disease (representative predictors of worse outcomes in COVID-19) in patients with pre-existing AF. Patients with NOAF were characterized by a worse inflammatory status during the hospital course than the patients in the other groups. The NOAF group had higher levels of CRP and LDH at the time of admission, and the incidence of ARDS and sepsis was more common in patients with NOAF than in those with pre-existing AF and SR. These findings may support the hypothesis that the incidence of NOAF in patients with COVID-19 is mainly based on cardiac injury due to systemic inflammation, and stimulated sympathetic nervous system due to unstable hemodynamics. ${ }^{28-30}$ Our results also support that severe inflammation is responsible for new-onset CVD after SARS-CoV-2 infection, especially NOAF.

In general, higher levels of CRP, reflecting critically ill conditions in infected patients, are related to the incidence of cardiac arrhythmia, which is represented by AF. ${ }^{31}$ The development of cardiac arrhythmia is caused by several factors, including baseline arrhythmogenic substrate, stimulated autonomic nervous system, and inflammation;28 thus, the occurrence of cardiac arrhythmia is associated with worse clinical outcomes in a critically ill population. ${ }^{31,32}$ In particular, unstable activity of the autonomic nervous system resulting from systemic inflammation has a significant role in the development of cardiac arrhythmia. ${ }^{29,30}$ These relationships between inflammation status and the incidence of NOAF may be explained by increased oxidative damage to the fibrous atrium. ${ }^{33,34}$ Our results showed that the NOAF group had higher levels of CRP and LDH at the time of admission, as well as a higher rate of ARDS, highlighting the relationship between inflammatory status and NOAF incidence. It has been debated and reported that SARS-CoV-2 infection would cause newonset cardiovascular diseases, ${ }^{35}$ although a recent report demonstrated that the incidence rate of NOAF is not specifically higher in patients with COVID-19, and that the total rate of NOAF is comparable to that of influenza.20 This fact may support the hypothesis that the occurrence of AF during COVID-19 infection is a generalized response 
to systemic inflammation as a result of severe viral illness.

Previous studies have reported that COVID-19 is a risk factor for both arterial and venous thrombotic events. ${ }^{36-38}$ In addition, the incidence of AF is a cause of embolic events, including stroke, as a result of left atrial thrombosis. ${ }^{39}$ Based on these facts, pre-existing AF and NOAF in patients with COVID-19 is presumed to be a notable predictor of thrombotic events, whereas a recent report suggested that NOAF in patients with COVID-19 was an independent predictor of embolic events. ${ }^{40}$ Consistently, we found that the NOAF group had a higher prevalence of stroke events than the pre-existing AF and SR groups. This is despite the fact that the pre-existing AF group had more comorbidities such as heart failure and chronic kidney disease, which is a classical risk factor for thrombotic events and higher CHADS2-VASc scores. The mechanism underlying the higher rate of stroke in patients with NOAF remains unclear, and there is a close relationship between the severity of COVID-19 and thrombotic events. ${ }^{36-38}$ Thus, the development of stroke in NOAF may result mainly from thrombotic tendencies in patients severe COVID-19. ${ }^{\mathbf{4 1}, 42}$ It is also noteworthy that the proportion of prior oral anticoagulation was lower in patients with NOAF than in those with pre-existing AF (pre-existing AF vs. NOAF: $60.0 \%$ vs. $3.6 \%, \mathrm{P}<0.001$ ). The relationship between prior oral anticoagulation therapy and the incidence of stroke after admission is unclear; hence, this possibility should be investigated in future studies.

After hospitalization, NOAF was associated with a higher incidence of ARDS, sepsis, septic shock, acute kidney injury, and multiple organ diseases in patients with CVDRF. Additionally, we found that patients with NOAF after SARS-CoV-2 infection had higher mortality rates. The effect of NOAF on the occurrence of these adverse events was unclear, although our data support that the incidence of NOAF is a beneficial predictor of in-hospital outcomes of patients with COVID-19. Furthermore, a recent report suggested that the incidence of NOAF in the general population hospitalized with COVID-19 is strongly associated with higher in-hospital mortality. ${ }^{20}$ Therefore, the incidence of NOAF may be a useful predictor of clinical outcomes regardless of the presence of CVDRF. In some cases, detecting accurate severity is difficult according to "silent hypoxemia";43,44 thus, the incidence of NOAF, which is an easily accessible predictor, would be useful in the management of COVID-19.

\section{Study Limitations}

With regard to limitations, this was a retrospective study with missing baseline characteristics. In addition, the incidence of NOAF was also collected retrospectively from medical records; thus, it is possible to overlook its incidence during hospitalization. The missing data may lead to varying results regarding the effect of NOAF and preexisting AF on outcomes. Moreover, the date of onset of NOAF was not collected in the CLAVIS-COVID registry, the duration between the occurrence of NOAF and inhospital events could not be assessed, and the information about systemic condition at the time of the incidence of NOAF was unclear. Therefore, the duration and relationships between the incidence of NOAF and other in-hospital events were not analyzed. Further studies are needed to address these concerns. As we retrospectively analyzed the effect of NOAF, there may be an immortal time bias regarding the association between the incidence of NOAF and the outcomes. The study population of the CLAVISCOVID registry was enrolled from January 2020 to May 2020, which was the first wave of the COVID-19 pandemic in Japan, thus our cohort did not include the specific individuals with COVID-19 that have a mutation of virus strains. In addition, given several treatment options established as of 2021, it is unclear whether the effect of NOAF on outcomes is comparable in the population after 2021. Further research is needed to focus on these limitations.

\section{Conclusions}

In patients with COVID-19 and CVDRF, the NOAF group was independently associated with worse clinical outcomes than the pre-existing AF and SR groups. These patients are at a high risk of adverse events during hospitalization; hence, intensive monitoring and treatment strategies are needed.

\section{Acknowledgments}

We wish to acknowledge all investigators who participated in CLAVISCOVID registry, the staff of the Department of Cardiology at the Ebara Hospital who worked hard to input the data, and the Japanese Circulation Society.

\section{Disclosures}

T.Y. is associated with endowed departments of Abbott Vascular Japan, Boston Scientific Japan, Japan Lifeline, WIN International, and Takeyama KK. S. Kohsaka received unrestricted research grants from the Department of Cardiology, Keio University School of Medicine provided by Daiichi Sankyo Co., Ltd. and Bristol-Meyers Squibb, and lecture fees from AstraZeneca and Bristol-Meyers Squibb. Y.M. is affiliated with a department endowed by Philips Respironics, ResMed, Teijin Home Healthcare, and Fukuda Denshi received an honorarium from Otsuka Pharmaceutical Co. and Novartis Japan, received a consultant fee from Otsuka Pharmaceutical Co., and joint research funds from Otsuka Pharmaceutical Co. and Pfizer Inc. T.I., K.N. are members of Circulation Journal's Editorial Team.

\section{IRB Information}

The investigation conformed to the principles outlined in the Declaration of Helsinki. The study protocol, including the use of an opt-out consent method, was approved by the ethics committee of Toho University Omori Medical Center (No. M20253) and the local ethics committees of all participating institutions. Furthermore, this clinical study was registered with the University Hospital Medical Information Network Clinical Trial Registry (UMIN-ID: UMIN000040598) before the first patient was enrolled, in accordance with the International Committee of Medical Journal Editors.

\section{Data Availability}

Due to the nature of this research, the participants of this study did not agree for their data to be shared publicly or upon request. Hence, the data are not available.

\section{References}

1. Ip RJ, Ali A, Baloch ZQ, Al-Abcha A, Jacob C, Arnautovic J, et al. Atrial fibrillation as a predictor of mortality in high risk COVID-19 patients: A multicentre study of 171 patients. Heart Lung Circ 2021; 30: $1151-1156$.

2. Wu C, Chen X, Cai Y, Xia J, Zhou X, Xu S, et al. Risk factors associated with acute respiratory distress syndrome and death in patients with coronavirus disease 2019 pneumonia in Wuhan, China. JAMA Intern Med 2020; 180: 934-943.

3. Sandoval Y, Januzzi JL Jr, Jaffe AS. Cardiac troponin for assessment of myocardial injury in COVID-19: JACC review topic of the week. J Am Coll Cardiol 2020; 76: 1244-1258.

4. Lala A, Johnson KW, Russak AJ, Paranjpe I, Zhao S, Solani S, et al. Prevalence and impact of myocardial injury in patients hospitalized with COVID-19 infection. medRxiv, doi:10.1101/20 
20.04.20.20072702

5. Lombardi CM, Carubelli V, Iorio A, Inciardi RM, Bellasi A, Canale C, et al. Association of troponin levels with mortality in Italian patients hospitalized with coronavirus disease 2019: Results of a multicenter study. JAMA Cardiol 2020; 5: 1274 1280 .

6. Knight SR, Ho A, Pius R, Buchan I, Carson G, Drake TM, et al Risk stratification of patients admitted to hospital with covid-19 using the ISARIC WHO Clinical Characterisation Protocol: Development and validation of the $4 \mathrm{C}$ Mortality Score. $B M J$ 2020; 370: m3339.

7. Ali R, Qayyum F, Ahmed N, Haroon MZ, Irshad R, Sajjad S, et al. Isaric $4 \mathrm{c}$ mortality score as a predictor of in-hospital mortality in Covid-19 patients admitted in Ayub teaching hospital during first wave of the pandemic. J Ayub Med Coll Abbottabad 2021; 33: $20-25$.

8. Bassand JP, Accetta G, Camm AJ, Cools F, Fitzmaurice DA, Fox KA, et al. Two-year outcomes of patients with newly diagnosed atrial fibrillation: Results from GARFIELD-AF. Eur Heart J 2016; 37: 2882-2889.

9. Schnabel RB, Yin X, Gona P, Larson MG, Beiser AS, McManus $\mathrm{DD}$, et al. 50 year trends in atrial fibrillation prevalence, incidence, risk factors, and mortality in the Framingham Heart Study: A cohort study. Lancet 2015; 386: 154-162.

10. Mountantonakis SE, Saleh M, Fishbein J, Gandomi A, Lesser $\mathrm{M}$, Chelico J, et al. Atrial fibrillation is an independent predictor for in-hospital mortality in patients admitted with SARS-CoV-2 infection. Heart Rhythm 2021; 18: 501-507.

11. Wetterslev M, Jacobsen PK, Hassager C, Jøns C, Risum N, Pehrson S, et al. Cardiac arrhythmias in critically ill patients with coronavirus disease 2019: A retrospective population-based cohort study. Acta Anaesthesiol Scand 2021; 65: 770-777.

12. Kang Y, Chen T, Mui D, Ferrari V, Jagasia D, Scherrer-Crosbie $\mathrm{M}$, et al. Cardiovascular manifestations and treatment considerations in COVID-19. Heart 2020; 106: 1132-1141.

13. Siripanthong B, Nazarian S, Muser D, Deo R, Santangeli P, Khanji MY, et al. Recognizing COVID-19-related myocarditis: The possible pathophysiology and proposed guideline for diagnosis and management. Heart Rhythm 2020; 17: 1463-1471.

14. Bader F, Manla Y, Atallah B, Starling RC. Heart failure and COVID-19. Heart Fail Rev 2021; 26: 1-10.

15. Tomasoni D, Italia L, Adamo M, Inciardi RM, Lombardi CM, Solomon SD, et al. COVID-19 and heart failure: From infection to inflammation and angiotensin II stimulation. Searching for evidence from a new disease. Eur J Heart Fail 2020; 22: 957-966.

16. Bhatla A, Mayer MM, Adusumalli S, Hyman MC, Oh E, Tierney A, et al. COVID-19 and cardiac arrhythmias. Heart Rhythm 2020; 17: 1439-1444.

17. Harrison SL, Fazio-Eynullayeva E, Lane DA, Underhill P, Lip GYH. Atrial fibrillation and the risk of 30-day incident thromboembolic events, and mortality in adults $\geq 50$ years with COVID- 19 . J Arrhythm 2021; 37: 231-237.

18. Klok FA, Kruip MJHA, van der Meer NJM, Arbous MS, Gommers DAMP, Kant KM, et al. Incidence of thrombotic complications in critically ill ICU patients with COVID-19. Thromb Res 2020; 191: 145-147.

19. Ergun B, Ergan B, Sozmen MK, Kucuk M, Yakar MN, Comert $\mathrm{B}$, et al. New-onset atrial fibrillation in critically ill patients with coronavirus disease 2019 (COVID-19). J Arrhythm 2021; 37: $1196-1204$.

20. Musikantow DR, Turagam MK, Sartori S, Chu E, Kawamura I, Shivamurthy P, et al. Atrial fibrillation in patients hospitalized with COVID-19: Incidence, predictors, outcomes, and comparison to influenza. JACC Clin Electrophysiol 2021; 7: 1120-1130.

21. Grasselli G, Greco M, Zanella A, Albano G, Antonelli M, Bellani $\mathrm{G}$, et al. Risk factors associated with mortality among patients with COVID-19 in intensive care units in Lombardy, Italy. JAMA Intern Med 2020; 180: 1345-1355.

22. Parohan M, Yaghoubi S, Seraji A, Javanbakht MH, Sarraf P, Djalali M. Risk factors for mortality in patients with Coronavirus disease 2019 (COVID-19) infection: A systematic review and meta-analysis of observational studies. Aging Male 2020; 23: $1416-1424$.

23. Zheng Z, Peng F, Xu B, Zhao J, Liu H, Peng J, et al. Risk factors of critical \& mortal COVID-19 cases: A systematic literature review and meta-analysis. $J$ Infect 2020; 81: e16-e25.

24. Matsumoto S, Kuroda S, Sano T, Kitai T, Yonetsu T, Kohsaka $\mathrm{S}$, et al. Clinical and biomarker profiles and prognosis of elderly patients with coronavirus disease 2019 (COVID-19) with cardiovascular diseases and/or risk factors. Circ J 2021; 85: 921-928.

25. The International Severe Acute Respiratory and Emerging Infection Consortium (ISARIC). Clinical Data Collection: The COVID-19 Case Report Forms (CRFs). https://isaric.org/ research/covid-19-clinical-research-resources/covid-19-crf/ (accessed December 1, 2020).

26. World Health Organization. Clinical management of COVID19. https://apps.who.int/iris/bitstream/handle/10665/332196/ WHO-2019-nCoV-clinical-2020.5-eng.pdf (accessed June 1, 2020).

27. Kuroda S, Matsumoto S, Sano T, Kitai T, Yonetsu T, Kohsaka $\mathrm{S}$, et al. External validation of the $4 \mathrm{C}$ Mortality Score for patients with COVID-19 and pre-existing cardiovascular diseases/risk factors. BMJ Open 2021; 11: e052708.

28. Aldhoon B, Melenovsky V, Peichl P, Kautzner J. New insights into mechanisms of atrial fibrillation. Physiol Res 2010; 59: 1-12.

29. Kuhlkamp V, Schirdewan A, Stangl K, Homberg M, Ploch M, Beck OA. Use of metoprolol CR/XL to maintain sinus rhythm after conversion from persistent atrial fibrillation: A randomized, double-blind, placebo-controlled study. J Am Coll Cardiol 2000; 36: $139-146$.

30. Maisel WH, Rawn JD, Stevenson WG. Atrial fibrillation after cardiac surgery. Ann Intern Med 2001; 135: 1061-1073.

31. Klein Klouwenberg PM, Frencken JF, Kuipers S, Ong DS, Peelen LM, van Vught LA, et al. Incidence, predictors, and outcomes of new-onset atrial fibrillation in critically ill patients with sepsis. A cohort study. Am J Respir Crit Care Med 2017; 195: 205-211.

32. Salman S, Bajwa A, Gajic O, Afessa B. Paroxysmal atrial fibrillation in critically ill patients with sepsis. J Intensive Care Med 2008; 23: $178-183$.

33. Frustaci A, Chimenti C, Bellocci F, Morgante E, Russo MA, Maseri A. Histological substrate of atrial biopsies in patients with lone atrial fibrillation. Circulation 1997; 96: 1180-1184.

34. Mihm MJ, Yu F, Carnes CA, Reiser PJ, McCarthy PM, Van Wagoner DR, et al. Impaired myofibrillar energetics and oxidative injury during human atrial fibrillation. Circulation 2001; 104: $174-180$.

35. Clerkin KJ, Fried JA, Raikhelkar J, Sayer G, Griffin JM, Masoumi A, et al. COVID-19 and cardiovascular disease. Circulation 2020; 141: 1648-1655.

36. Zhang L, Feng X, Zhang D, Jiang C, Mei H, Wang J, et al. Deep vein thrombosis in hospitalized patients with COVID-19 in Wuhan, China: Prevalence, risk factors, and outcome. Circulation 2020; 142: $114-128$

37. Ali MAM, Spinler SA. COVID-19 and thrombosis: From bench to bedside. Trends Cardiovasc Med 2021; 31: 143-160.

38. Price LC, McCabe C, Garfield B, Wort SJ. Thrombosis and COVID-19 pneumonia: The clot thickens! Eur Respir J 2020; 56: 2001608.

39. Wolf PA, Dawber TR, Thomas HE Jr, Kannel WB. Epidemiologic assessment of chronic atrial fibrillation and risk of stroke: The Framingham study. Neurology 1978; 28: 973-977.

40. Pardo Sanz A, Salido Tahoces L, Ortega Pérez R, González Ferrer E, Sánchez Recalde A, Zamorano Gómez JL. New-onset atrial fibrillation during COVID-19 infection predicts poor prognosis. Cardiol J 2021; 28: 34-40.

41. Llitjos JF, Leclerc M, Chochois C, Monsallier JM, Ramakers M, Auvray M, et al. High incidence of venous thromboembolic events in anticoagulated severe COVID-19 patients. J Thromb Haemost 2020; 18: $1743-1746$.

42. Fox SE, Akmatbekov A, Harbert JL, Li G, Quincy Brown J, Vander Heide RS. Pulmonary and cardiac pathology in African American patients with COVID-19: An autopsy series from New Orleans. Lancet Respir Med 2020; 8: 681-686.

43. Tobin MJ, Laghi F, Jubran A. Why COVID-19 silent hypoxemia is baffling to physicians. Am J Respir Crit Care Med 2020; 202: $356-360$.

44. Rahman A, Tabassum T, Araf Y, Al Nahid A, Ullah MA, Hosen MJ. Silent hypoxia in COVID-19: Pathomechanism and possible management strategy. Mol Biol Rep 2021; 48: 3863-3869. 